\title{
Presymplectic Geometry and Fermat's \\ Principle for anisotropic media
}

\author{
J.F. CARIÑNEn**, AND J. NASARRE $\S \S$ \\ *Depto. Física Teórica, Univ. de Zaragoza, 50009 Zaragoza, Spain. \\ $\S \S$ Seminario de Matemáticas, IES Miguel Catalán, 50009 Zaragoza, Spain.
}

\begin{abstract}
The tools of presymplectic geometry are used to study light rays trajectories in anisotropic media.
\end{abstract}

The usefulness of the Lie-Hamilton optics in many different problems as ray tracing for ray design and computation of aberrations suggests the study of what happens for anisotropic media, because of the recent interest in the use of anisotropic optical material. This motivated a very recent paper [1] where the Hamiltonian formulation of geometric anisotropic optics was studied. The theory was reexamined in [2]. The basic principle of the theory is the celebrated Fermat's principle of least time (or extremal time if reflection is also allowed). In other words, light rays connecting points $A$ and $B$ are lines in the space in such a way that they satisfy the following variational condition:

$$
\delta \int_{A}^{B} n d s=0 .
$$

The refractive index of the medium is given by the quotient $n=\frac{c}{v}$, and then physics tell us that $n>1$.

In a recent paper [3] we analysed from a geometric perspective the relationship for the case of isotropic media of the problem of determination of extremal curves for (11) with that of geodesics of a Riemannian metric conformal to the Euclidean metric. We will consider here the case in which the medium is not isotropic but it may depend on the velocity, or more specifically, on the direction of the ray. In this last case the problem cannot be reduced to a problem of Riemannian geometry as it happened when the refractive index $n$ only depended on the position. So, the techniques of Presymplectic geometry are unavoidable for dealing with this dependence of the refractive index with the ray direction.

To begin we remark the strong similarity of Fermat's principle with the more traditional Hamilton's principle of Classical Mechanics, with a Lagrangian function given by

$$
L=n \sqrt{g(v, v)} .
$$


This Lagrangian function is differentiable only in the set of velocity phase space obtained by removing the null velocity points, i.e., the zero section of the tangent bundle. Moreover, the Lagrangian $L$ is homogeneus of degree one,

$$
v^{i} \frac{\partial L}{\partial v^{i}}=L
$$

and consequently the corresponding energy function vanishes identically. Therefore the Lagrangian is singular, because taking derivatives with respect to $v^{j}$ of both sides of the preceding equation we obtain

$$
\frac{\partial^{2} L}{\partial v^{i} \partial v^{j}} v^{i}=0
$$

and then the Hessian matrix

$$
W_{i j}=\frac{\partial^{2} L}{\partial v^{i} \partial v^{j}}
$$

is singular. The theory should be carefully reexamined using the tools of Presymplectic Geometry, as it was done in [3] for the isotropic media, where the tools of Riemannian Geometry were shown to be very useful. Actually, it is possible to show that in the latter case the solution curves for the regular Lagrangian system described by the regular Lagrangian

$$
\mathbb{L}=\frac{1}{2} n^{2} g(v, v)
$$

are just the curves solution of the original problem, even if the curves are reparametrized (see e.g. [4 and references therein). Our aim is to analyse what happens in the more general case in which the refractive index can depend on the ray direction, i.e., the refractive index is a homogeneous function of degree zero of velocities in the set obtained by removing the zero section of the velocity phase espace.

The geometric approach to Lagrangian Classical mechanics uses as velocity phase space the tangent bundle $T M$ of the configuration space $M$ that is assumed to be a differentiable manifold of dimension $N$. ¿From now on we will follow the notation used in [5]. The tangent structure is characterized by a $(1,1)$-tensor field called vertical endomorphism $S$ that in terms of natural coordinates $\left(q^{i}, v^{i}\right)$ of the tangent bundle $T M$ is given by

$$
S=\frac{\partial}{\partial v^{i}} \otimes d q^{i}
$$

Given a function $L \in C^{\infty}(T M)$, we may define an exact 2 -form in $T M, \omega_{L}=-d \theta_{L}$, with the 1 -form $\theta_{L}$ being defined by $\theta_{L}=d L \circ S$, and a function $E_{L}=\Delta(L)-L$, called energy function. In the above mentioned coordinates of $T M$ we have the following expressions:

$$
\begin{aligned}
\theta_{L} & =\frac{\partial L}{\partial v^{i}} d q^{i} \\
\omega_{L} & =\frac{\partial^{2} L}{\partial q^{i} \partial v^{j}} d q^{j} \wedge d q^{i}+\frac{\partial^{2} L}{\partial v^{i} \partial v^{j}} d q^{i} \wedge d v^{j} \\
\Delta & =v^{i} \frac{\partial}{\partial v^{i}} \\
E_{L} & =v^{i} \frac{\partial L}{\partial v^{i}}-L
\end{aligned}
$$


Here $\Delta \in \mathfrak{X}(T M)$ denotes the Liouville vector field generating dilations along the fibres. When $\omega_{L}$ is nondegenerate, i.e., the Hessian matrix (5) is regular, it defines a symplectic structure on $T M$, and a vector field $\Gamma_{L}$ uniquely determined by $i\left(\Gamma_{L}\right) \omega_{L}=d E_{L}$.

We will next examine the problem of light rays, even for the more general case in which the refractive index $n$ depends on the ray direction. This means that the refractive index must be homogeneous of degree zero in the velocities, $\Delta n=0$. We define a new Lagrangian $\mathbb{L}=\frac{1}{2} L^{2}$. The Lagrangian $\mathbb{L}$ is homogeneous of degree two in the velocities, $\Delta \mathbb{L}=2 \mathbb{L}=L^{2}$, and then $E_{\mathbb{L}}=\mathbb{L}$.

Now taking into account that $\theta_{\mathbb{L}}=d \mathbb{L} \circ S$ we see that the Liouville 1 -form $\theta_{\mathbb{L}}$ is proportional to $\theta_{L}$, namely, $\theta_{\mathbb{L}}=L \theta_{L}$, (see [6]) and then

$$
\omega_{L}=\frac{1}{L} \omega_{\mathbb{L}}+\frac{1}{L^{3}} d \mathbb{L} \wedge \theta_{\mathbb{L}}
$$

As indicated above, when the refractive index $n$ does not depend on velocities the 2 -form $\omega_{\mathbb{L}}$ is regular. In this more general case, however, it may be singular, because

$$
\omega_{\mathbb{L}}=L \omega_{L}-d L \wedge \theta_{L}
$$

means that

$$
\omega_{\mathbb{L}}^{\wedge N}=L^{N} \omega_{L}^{\wedge N}-N L^{N-1} d L \wedge \theta_{L} \wedge \omega_{L}^{\wedge(N-1)}=-N L^{N-1} d L \wedge \theta_{L} \wedge \omega_{L}^{\wedge(N-1)}
$$

and then $\omega_{\mathbb{L}}^{\wedge N}$ can be identically null and in this case $\mathbb{L}$ would be singular.

In the following we will restrict ourselves to the case in which $\mathbb{L}$ is regular and therefore there will be a uniquely defined vector field $\Gamma_{\mathbb{L}}$ such that

$$
i\left(\Gamma_{\mathbb{L}}\right) \omega_{\mathbb{L}}=d E_{\mathbb{L}}=d \mathbb{L}
$$

and then

$$
i\left(\Gamma_{\mathbb{L}}\right)(d \mathbb{L})=\Gamma_{\mathbb{L}}(\mathbb{L})=0 .
$$

Moreover, $\Gamma_{\mathbb{L}}$ is a second order differential equation vector field.

First we check that the Liouville vector field $\Delta$ lies in $\operatorname{ker} \omega_{L}$. Indeed,

$$
i(\Delta) \omega_{L}=\frac{1}{L} i(\Delta) \omega_{\mathbb{L}}+\frac{1}{L^{3}} \Delta \mathbb{L} \theta_{\mathbb{L}}-\frac{1}{L^{3}} d \mathbb{L}\left(i(\Delta) \theta_{\mathbb{L}}\right)
$$

and taking into account that $\Delta$ is vertical and $\theta_{\mathbb{L}}$ semibasic, the last term vanishes. Moreover,

$$
i(\Delta) \omega_{\mathbb{L}}=-\mathcal{L}_{\Delta} \theta_{\mathbb{L}}+d\left(i(\Delta) \theta_{\mathbb{L}}\right)=-\mathcal{L}_{\Delta} \theta_{\mathbb{L}}=-\theta_{\mathbb{L}}
$$

and therefore, taking into account that $\Delta \mathbb{L}=2 \mathbb{L}=L^{2}$ we find that

$$
i(\Delta) \omega_{L}=0
$$

Secondly, $\Gamma_{\mathbb{L}}$ is also in the kernel of $\omega_{L}$, because

$$
i\left(\Gamma_{\mathbb{L}}\right) \omega_{L}=\frac{1}{L} i\left(\Gamma_{\mathbb{L}}\right) \omega_{\mathbb{L}}+\frac{1}{L^{3}}\left(\Gamma_{\mathbb{L}} \mathbb{L}\right) \theta_{\mathbb{L}}-\frac{1}{L^{3}} d \mathbb{L}\left[i\left(\Gamma_{\mathbb{L}}\right) \theta_{\mathbb{L}}\right]
$$


and $\Gamma_{L}$ being a SODE,

$$
i\left(\Gamma_{\mathbb{L}}\right) \theta_{\mathbb{L}}=(d \mathbb{L} \circ S)\left(\Gamma_{\mathbb{L}}\right)=\Delta \mathbb{L}=2 \mathbb{L}=L^{2}
$$

and therefore

$$
i\left(\Gamma_{\mathbb{L}}\right) \omega_{L}=0 .
$$

Finally under the assumption that $\mathbb{L}$ is regular, $\operatorname{ker} \omega_{L}$ is generated by $\Delta$ and $\Gamma_{\mathbb{L}}$. Indeed, given a vertical vector field $V \in \operatorname{ker} \omega_{L}$, then,

$$
0=i(V) \omega_{L}=\frac{1}{L} i(V) \omega_{\mathbb{L}}+\frac{1}{L^{3}} V(\mathbb{L}) \theta_{\mathbb{L}}
$$

and in particular

$$
\frac{1}{L} i(\Delta) \omega_{\mathbb{L}}=-\frac{1}{L^{3}} \Delta(\mathbb{L}) \theta_{\mathbb{L}}=-\frac{1}{L} \theta_{\mathbb{L}}
$$

and therefore

$$
i(V) \omega_{\mathbb{L}}=\frac{V(\mathbb{L})}{L^{2}} i(\Delta) \omega_{\mathbb{L}}
$$

and as $\omega_{\mathbb{L}}$ is assumed to be regular, $V$ should be proportional to $\Delta$. Then, $\operatorname{dim} V\left(\operatorname{ker} \omega_{L}\right)=1$ and because of the relation $\operatorname{dim}\left(\operatorname{ker} \omega_{L}\right) \leq 2 \operatorname{dim} V\left(\operatorname{ker} \omega_{L}\right)$ (see [7]), we can conclude that $\operatorname{dim}\left(\operatorname{ker} \omega_{L}\right)=2$.

Under these circumstances is possible to apply the reduction theory of presymplectic manifolds, following the ideas developed by Marsden and Weinstein [8]. Presymplectic structures may arise either when using some constants of motion for reducing the phase space or also when the Lagrangian that has been chosen is singular. Then we will have a pair $\left(P_{0}, \Omega_{0}\right)$ where $\Omega_{0}$ is a closed but degenerate $2-$ form. A consistent solution of the dynamical equation can only be found in some points, leading in this way to the final constraint submanifold $P$ introduced by Dirac (see e.g. [9]). The pull back $\Omega$ of the form $\Omega_{0}$ on this manifold will be assumed to be of constant rank. The recipe for dealing with these systems was given by Marsden and Weinstein [8]. First, in every point $m \in P$, $\operatorname{ker} \Omega_{m}$ is a $k$-dimensional linear space, so defining what is called a $k$-dimensional distribution. The important point is that closedness of $\Omega$ is enough to warrant that the distribution is integrable (and then it is called foliation): for any point $m \in P$, there is a $k$-dimensional submanifold of $P$ passing through $m$ and such that the tangent space at any point $m^{\prime}$ of this surface coincides with ker $\Omega_{m^{\prime}}$. Such integral $k$-dimensional submanifolds give a foliation of $P$ by disjoint leaves and in the case in which the quotient space $\widetilde{P}=P / \operatorname{ker} \Omega$ is a differentiable manifold, then it is possible to define a nondegenerate closed 2 -form $\widetilde{\Omega}$ in $\widetilde{P}$ such that $\widetilde{\pi}^{*} \widetilde{\Omega}=\Omega$. Here $\widetilde{\pi}: P \rightarrow \widetilde{P}$ is the natural projection. It suffices to define $\widetilde{\Omega}\left(\widetilde{v}_{1}, \widetilde{v}_{2}\right)=\Omega\left(v_{1}, v_{2}\right)$, where $v_{1}$ and $v_{2}$ are tangent vectors to $P$ projecting under $\widetilde{\pi}_{*}$ onto $\widetilde{v}_{1}$ and $\widetilde{v}_{2}$ respectively. The symplectic space $(\widetilde{P}, \widetilde{\Omega})$ is said to be the reduced space. We will illustrate the method finding coordinates adapted to the distribution defined by the kernel ker $\omega_{L}$ of the presymplectic structure defined by the singular optical Lagrangian in the case of a system in which either the index $n$ depends on the third coordinate $x^{3}$ alone or the very interesting case in which the system is anisotropic and $n$ is a function of the ray direction. We will determine the quotient reduced space and we will look for Darboux coordinates in this reduced symplectic manifold. Once Darboux coordinates have been found we can consider the problem from the active viewpoint and take advantage of the algebraic methods recently developed for computing aberrations (see e.g. 110). 
Let us now consider the most general isotropic case in which the refractive index of the medium is not constant but it is given by a smooth function $n\left(x^{1}, x^{2}, x^{3}\right)$. Fermat's principle suggests us to consider the corresponding mechanical problem described by a singular Lagrangian $L(q, v)=[g(v, v)]^{1 / 2}$, where $g$ is a metric conformal to the Euclidean metric $g_{0}$,

$$
g(v, w)=n^{2} g_{0}(v, w)
$$

This problem was analysed in [6] where, as above is cited, it was shown that its study can be reduced to that of a regular Lagrangian $\mathbb{L}=\frac{1}{2} L^{2}$. This Lagrangian $\mathbb{L}$ is quadratic in velocities and the dynamical vector field $\Gamma_{\mathbb{L}}$ solution of the dynamical equation $i\left(\Gamma_{\mathbb{L}}\right) \omega_{\mathbb{L}}=$ $d E_{\mathbb{L}}=d \mathbb{L}$ is not only a second order differential equation vector field but, moreover, it is a spray [11], the projection onto $\mathbb{R}^{3}$ of its integral curves being the geodesics of the Levi-Civita connection defined by $g$. Then, $\Gamma_{\mathbb{L}}$ is the geodesic spray given by

$$
\Gamma_{\mathbb{L}}=v^{i} \frac{\partial}{\partial q^{i}}-\Gamma_{j k}^{i} v^{j} v^{k} \frac{\partial}{\partial v^{i}}
$$

where the Christoffel symbols $\Gamma^{i}{ }_{j k}$ are

$$
\Gamma_{j k}^{i}=\frac{1}{2} g^{i l}\left[\frac{\partial g_{k l}}{\partial q^{j}}+\frac{\partial g_{j l}}{\partial q^{k}}-\frac{\partial g_{j k}}{\partial q^{l}}\right]
$$

with $g^{i j}$ being the inverse matrix of $g_{i j}$.

In the particular case we are considering where $g(v, w)=n^{2} g_{0}(v, w)$, it was also shown above that the kernel of $\omega_{L}$ is two-dimensional and it is generated by $\Gamma_{\mathbb{L}}$ and the Liouville vector field $\Delta$. The distribution defined by ker $\omega_{L}$ is integrable because $\omega_{L}$ is closed; actually $\left[\Delta, \Gamma_{\mathbb{L}}\right]=\Gamma_{\mathbb{L}}$ and the distribution is also generated by $\Delta$ and $K$ defined by $K=\frac{1}{v^{3}} \Gamma_{\mathbb{L}}$, for which $[\Delta, K]=0$. In cartesian coordinates the Christoffel symbols are expressed as follows:

$$
\Gamma_{j k}^{i}=\frac{1}{n}\left[\frac{\partial n}{\partial x^{j}} \delta_{k}^{i}+\frac{\partial n}{\partial x^{k}} \delta_{j}^{i}-\frac{\partial n}{\partial x^{i}} \delta_{k}^{j}\right] .
$$

and the vector field $K$ is given as

$$
K=\frac{1}{v^{3}}\left[v^{i} \frac{\partial}{\partial x^{i}}-\left(\frac{2}{n} v^{i}(v \cdot \nabla n)-\frac{\|v\|^{2}}{n} \frac{\partial n}{\partial x^{i}}\right) \frac{\partial}{\partial v^{i}}\right] .
$$

The theory of distributions suggests us the introduction of new local coordinates $y^{i}=$ $F^{i}(x, v), i=1, \ldots, 6$, adapted to the distribution defined by $\operatorname{ker} \omega_{L}$, i.e., such that $K=\frac{\partial}{\partial y^{3}}$, $\Delta=\frac{\partial}{\partial y^{6}}$ (see [12]). The search for these new coordinates is based on the solution of the partial differential equation system

$$
K F^{1}=1, \quad \Delta F^{1}=0, \quad K F^{2}=0, \quad \Delta F^{2}=1,
$$

and

$$
K F^{2+a}=0, \quad \Delta F^{2+a}=0, \text { for } a=1 \ldots, 4 .
$$


The explicit computation of these functions depends very much on the choice of the function $n\left(x^{1}, x^{2}, x^{3}\right)$. We will illustrate next the theory with an particular example. If $n$ only depends on $x^{3}$, the presymplectic form can be written in the way

$$
\begin{aligned}
\omega_{L} & =d x^{1} \wedge d\left(\frac{n v^{1}}{\sqrt{v^{1^{2}}+v^{2^{2}}+v^{3^{2}}}}\right)+d x^{2} \wedge d\left(\frac{n v^{2}}{\sqrt{v^{1^{2}}+v^{2^{2}}+v^{3^{2}}}}\right) \\
& +\frac{n v^{3^{2}}}{\left(v^{1^{2}}+v^{2}+v^{3^{2}}\right)^{3 / 2}}\left[v^{1} d\left(\frac{v^{1}}{v^{3}}\right) \wedge d x^{3}+v^{2} d\left(\frac{v^{2}}{v^{3}}\right) \wedge d x^{3}\right],
\end{aligned}
$$

and the dynamical vector field is

$$
\begin{aligned}
\Gamma_{\mathbb{L}} & =v^{i} \frac{\partial}{\partial x^{i}}-\frac{2}{n} v^{1} v^{3} \frac{d n}{d x^{3}} \frac{\partial}{\partial v^{1}}-\frac{2}{n} v^{2} v^{3} \frac{d n}{d x^{3}} \frac{\partial}{\partial v^{2}} \\
& +\frac{1}{n}\left(v^{1^{2}}+v^{2^{2}}-v^{3^{2}}\right) \frac{d n}{d x^{3}} \frac{\partial}{\partial v^{3}} .
\end{aligned}
$$

After some calculations we find the solution of the former systems (see [3]). According to this, we will do the following choice for the new coordinates:

$$
\begin{aligned}
& y^{1}=x^{1}-\frac{v^{1}}{v^{2}} x^{2}, \\
& y^{2}=x^{2}-\int_{0}^{x^{3}} \frac{C_{3}}{\sqrt{\left(n^{2}(\zeta)-C_{3}^{2}\right)\left(1+C_{1}^{2}\right)}} d \zeta, \\
& y^{3}=x^{3} \\
& y^{4}=\frac{n v^{1}}{\sqrt{v^{1^{2}}+v^{2^{2}+v^{3}}}}, \\
& y^{5}=\frac{n v^{2}}{\sqrt{v^{1^{2}}+v^{2^{2}+v^{32}}}}, \\
& y^{6}=\log \left[n \sqrt{v^{1^{2}+v^{2}}+v^{3^{2}}}\right],
\end{aligned}
$$

where

$$
C_{1}=\frac{v^{1}}{v^{2}}
$$

and

$$
C_{3}=n \sqrt{\frac{v^{1^{2}}+v^{2^{2}}}{v^{1^{2}}+v^{2^{2}}+v^{3^{2}}}},
$$

doing the inverse change and after some easy calculations remains

$$
\widetilde{\omega}_{L}=d\left(y^{1}+\frac{y^{4}}{y^{5}} y^{2}\right) \wedge d y^{4}+d y^{2} \wedge d y^{5},
$$

which shows that

$$
\begin{aligned}
& \xi^{1}=y^{1}+\frac{y^{4}}{y^{5}} y^{2}=x^{1}-\frac{v^{1}}{v^{2}} \int_{0}^{x^{3}} \frac{C_{3}}{\sqrt{\left(n^{2}(\zeta)-C_{3}^{2}\right)\left(1+C_{1}^{2}\right)}} d \zeta, \\
& \xi^{2}=x^{2}-\int_{0}^{x^{3}} \frac{C_{3}}{\sqrt{\left(n^{2}(\zeta)-C_{3}^{2}\right)\left(1+C_{1}^{2}\right)}} d \zeta
\end{aligned}
$$


and the corresponding

$$
\eta^{1}=y^{4}=\frac{n v^{1}}{\sqrt{v^{1^{2}}+v^{2^{2}}+v^{3^{2}}}}, \quad \eta^{2}=y^{5}=\frac{n v^{2}}{\sqrt{v^{1^{2}}+v^{2^{2}}+v^{3^{2}}}}
$$

are Darboux coordinates for the symplectic form induced in the quotient space.

Let us now consider the particular but important case case in which the refractive index becomes constant out of a region. If for $x^{3}>L$, the index $n$ is constant, the above mentioned Darboux coordinates $\xi^{1}$ and $\xi^{2}$ are

$$
\xi^{1}=x^{1}-\frac{v^{1}}{v^{2}} \frac{C_{3} x^{3}}{\sqrt{\left(n^{2}-C_{3}^{2}\right)\left(1+C_{1}^{2}\right)}}, \quad \xi^{2}=x^{2}-\frac{C_{3} x^{3}}{\sqrt{\left(n^{2}-C_{3}^{2}\right)\left(1+C_{1}^{2}\right)}},
$$

up to a constant, and from the expresions of $C_{1}$ and $C_{3}$ we see that the Darboux coordinates become

$$
x^{1}-\frac{v^{1}}{v^{3}} x^{3}, \quad x^{2}-\frac{v^{2}}{v^{3}} x^{3}, \quad \frac{n v^{1}}{\sqrt{v^{1^{2}}+v^{2^{2}}+v^{3^{2}}}}, \quad \frac{n v^{2}}{\sqrt{v^{1^{2}}+v^{2^{2}}+v^{3^{2}}}},
$$

in full agreement with [13]. Therefore, for an optical system such that the refractive index depends only on $x^{3}$ and, furthermore, the region in which the index is not constant is bounded, we can choose Darboux coordinates by fixing a $x^{3}$ outside this region and taking Darboux coordinates for the corresponding problem of constant index. This justify the choice of coordinates as usually done for the ingoing and outgoing light rays in the corresponding constant index media, i.e. it shows the convenience of using flat screens in far enough regions on the left and right respectively, and then this change of Darboux coordinates seems to be, from an active viewpoint, a canonical transformation (see [14).

We will next find the symplectic structure arising in an anisotropic optical medium, as well as some Darboux coordinates for it. We recall what we are only considering anisotropic media for which the refractive index depends only on the ray direction, i.e., $n=n(v)$ but $\Delta n=0$. In this case the presymplectic form remains as

$$
\begin{aligned}
\omega_{L} & =\left[\|v\| \frac{\partial^{2} n}{\partial x^{j} \partial v_{i}}+\frac{v_{i}}{\|v\|} \frac{\partial n}{\partial x^{j}}\right] d x^{i} \wedge d x^{j} \\
& +\left[\|v\| \frac{\partial^{2} n}{\partial v_{j} \partial v_{i}}+\frac{v_{i}}{\|v\|} \frac{\partial n}{\partial v_{j}}+\frac{v_{j}}{\|v\|} \frac{\partial n}{\partial v_{i}}-\frac{n}{\|v\|^{3}} v_{i} v_{j}+\frac{n}{\|v\|} \delta_{j}^{i}\right] d x^{i} \wedge d v_{j}
\end{aligned}
$$

and the vector field associated with $\mathbb{L}$

$$
\Gamma_{\mathbb{L}}=v_{i} \frac{\partial}{\partial x^{i}}
$$

We still have that

$$
\left[\Delta, \Gamma_{\mathbb{L}}\right]=\Gamma_{\mathbb{L}}
$$

and then the ker $\omega_{L}$ defines an involutive, and hence an integrable, distribution that is also generated by $\Delta$ and $K, K$ being the vector field

$$
K=\frac{1}{v_{z}} \Gamma_{\mathbb{L}}
$$


commuting with $\Delta$. In this way, we can find new local coordinates adapted to the distribution that allow us to find later on the symplectic form in the quotient manifold, by solving the following differential equation systems:

$$
\begin{array}{ll}
\Delta f=0 & K f=0 . \\
\Delta f=1 & K f=0 . \\
\Delta f=0 & K f=1 .
\end{array}
$$

Acoording to the solution of the former systems we will do the following choice for the new coordinates:

$$
\begin{aligned}
& x_{1}=\frac{v_{x}}{v_{z}} z-x, \quad x_{2}=\frac{v_{y}}{v_{z}} z-y, \quad x_{3}=z \\
& y_{1}=\frac{v_{x}}{v_{z}}, \quad y_{2}=\frac{v_{y}}{v_{z}}, \quad y_{3}=\log v_{z},
\end{aligned}
$$

the inverse change being given by

$$
\begin{aligned}
x & =y_{1} x_{3}-x_{1}, \quad y=y_{2} x_{3}-x_{2}, \quad z=x_{3} \\
v_{x} & =y_{1} \exp y_{3}, \quad v_{y}=y_{2} \exp y_{3}, \quad v_{z}=\exp y_{3} .
\end{aligned}
$$

Moreover, in the reduction of the presymplectic form we must point out that the condition $\Delta n=0$ is written in the new coordinates as $\frac{\partial n}{\partial y_{3}}=0$. Finally, using the change of coordinate former, we get after some calculations the following symplectic form in the quotient manifold

$$
\begin{aligned}
\widetilde{\omega}_{L} & =d\left[\frac{n y_{1}}{\sqrt{y_{1}^{2}+y_{2}^{2}+1}}+\sqrt{y_{1}^{2}+y_{2}^{2}+1} \frac{\partial n}{\partial y_{1}}\right] \wedge d x_{1} \\
& +d\left[\frac{n y_{2}}{\sqrt{y_{1}^{2}+y_{2}^{2}+1}}+\sqrt{y_{1}^{2}+y_{2}^{2}+1} \frac{\partial n}{\partial y_{2}}\right] \wedge d x_{2}
\end{aligned}
$$

which is in full agreement with [13].

As a final comment, let us remark that, even in this case, if we restrict ourselves to a region of constant index, we recover the Darboux coordinates for constant index medium and we can think of the relation betwen the ingoing and outgoing light rays as a change of Darboux coordinates. Therefore this change of Darboux coordinates seems to be again, from an active viewpoint, a canonical transformation. The transformations of phase space will be, in general, non lineal, i.e., they generate optical aberrations. It is possible, finally, to analyse these aberrations using both group theoretical and Lie algebraic tools (see [10], [13], and references therein).

\section{References}

[1] Rivera A.L., Chumakov S.M. and Wolf K.B. 1994 Hamiltonian fundation of Geometrical anisotropic optics, Preprint I.I.M.A.S. 19

[2] Torres del Castillo G.F., 1995 Lagrangian and Hamiltonian Formulations of Geometrical Anisotropic Optics, Preprint Rev. Mex. Fis. (to appear) 
[3] Cariñena J.F. and Nasarre J., 1996 On the Symplectic structures arising in Geometric Optics, Forts. der Physik (to appear)

[4] Bazańanski S.L. and Jaranowski P., 1991 The Inverse Jacobi Problem, J. Phys. A: Math. Gen. 27, 3221-2234

[5] Cariñena J.F. and Rañada M.F., 1995 Comments on the Presymplectic formalism and the Theory of regular Lagrangians with constraints, J. Phys. A: Math. Gen. 28, L91-L97

[6] Cariñena J.F. and López C., 1991 Symplectic structure on the Set of Geodesics of a Riemannian Manifold, Int. J. Modern. Phys. A 6, 431-444

[7] Cariñena J.F. and Ibort L.A., 1985 Geometric Theory of the Equivalence of Lagrangians of Constrained Systems, J. Phys. A: Math. Gen. 18, 3335-41

[8] Marsden J.E. and Weinstein A., 1974 Reduction of symplectic manifolds with symmetry, Rep. Math. Phys. 5, 121-30

[9] Cariñena J.F., 1990 Theory of Singular Lagrangians, Forts. der Phys. 38, 641-680

[10] Dragt A.J. and Forest E., 1983 Computation of nonlinear behavior of Hamiltonian systems using Lie algebraic methods, J. Math. Phys. 24, 2734-2744

[11] Crampin M. and Pirani F.A.E., 1986 Applicable differential geometry (Cambridge U.P., London)

[12] Crampin M., 1983 Tangent bundle geometry for Lagrangian dynamics, J. Phys. A: Math. Gen. 16, 3775-3772

[13] Sánchez Mondragón J. and Wolf K.B., 1986 Lie Methods in Optics, Lect. Notes in Physics 250, (Springer)

[14] Guillemin V. and Sternberg S., 1984 Symplectic techniques in Physics, Cambridge Univ. Press. 